\title{
Stress testing in patients with chronic kidney disease: The need for ancillary markers for effective risk stratification and prognosis
}

\author{
Sripal Bangalore, MD, MHA, FACC, FAHA, FSCAI ${ }^{a}$ \\ ${ }^{a}$ Cardiac Catheterization Laboratory, Cardiovascular Outcomes Group, Cardiovascular Clinical \\ Research Center, The Leon H. Charney Division of Cardiology, New York University School of \\ Medicine, New York, NY
}

Received Aug 5, 2015; accepted Aug 5, 2015

doi:10.1007/s12350-015-0264-7

\section{See related article, pp. 560-569}

More than 20 million adults have chronic kidney disease (CKD) and nearly one-half million people have end-stage renal disease (ESRD) in the United States ${ }^{1}$ and with increasing prevalence of diabetes, obesity, and the elderly, this number is projected to increase exponentially by $2030 .^{2}$ Similarly, population studies in various countries indicate that CKD affects as many as 1 in 10 adults, or over 500 million people worldwide. ${ }^{3}$

Cardiovascular disease is the leading cause of morbidity and mortality among subjects with CKD, ${ }^{1,4,5} 15-30$ times higher than the age-adjusted cardiovascular mortality rate in the general population. ${ }^{6,7}$ The prognosis of patients with CKD and especially those with ESRD is poor and is worse than that for the general population who have cancers, heart failure, stroke, or acute myocardial infarction (MI), ${ }^{1}$ and as such patients with CKD are considered by some as coronary artery disease (CAD) risk equivalents. ${ }^{8,9}$ Although the prevalence of CAD is high, conventional cardiac risk factors such as hypertension, hyperlipidemia, smoking, or even the Framingham risk score are less predictive of CAD in CKD patients. ${ }^{10}$ Moreover, chest pain has poor sensitivity and specificity (65\% and 66\%, respectively) for predicting CAD in CKD

Reprint requests: Sripal Bangalore, MD, MHA, FACC, FAHA, FSCAI, Cardiac Catheterization Laboratory, Cardiovascular Outcomes Group, Cardiovascular Clinical Research Center, The Leon H. Charney Division of Cardiology, New York University School of Medicine, New York, NY 10016; sripalbangalore@gmail.com J Nucl Cardiol 2016;23:570-4.

$1071-3581 / \$ 34.00$

Copyright (C) 2015 American Society of Nuclear Cardiology. patients. ${ }^{11}$ As such stress testing is used as a modality to risk stratify patients with CKD or ESRD.

\section{STRESS TEST IN CKD/ESRD PATIENTS}

Although stress testing is used widely in patients with CKD/ESRD, there is controversy over the utility of stress testing when compared with invasive angiography as the best screening modality. ${ }^{12}$ Fundamental to this controversy is the low sensitivity of stress testing in the CKD/ESRD cohort compared with subjects without CKD (Table 1). ${ }^{13}$ Exercise electrocardiography (EKG) (treadmill or bicycle) is used in a minority of patients with CKD/ESRD as the prevalence of abnormal baseline EKG is high due to the presence of left ventricular hypertrophy, left bundle branch block, and ST-T changes that are either nonspecific or due to electrolyte abnormalities. Moreover, the exercise capacity of patients with CKD/ESRD is limited due to muscle fatigue, anemia, peripheral vascular disease, peripheral neuropathy, and other comorbidities. In addition, the sensitivity and specificity of exercise electrocardiography in CKD/ESRD patients is poor (Table 1). ${ }^{13,14}$

Similarly, the sensitivity and specificity of myocardial perfusion single photon emission computed tomography (SPECT) to detect CAD in CKD/ESRD patients are highly variable. In a prospective study of 138 patients referred for pre-renal transplant cardiac evaluation (43\% on dialysis) and underwent coronary artery calcium score, coronary computer tomography angiography, and SPECT, SPECT had a low sensitivity $(53 \%)$ and modest specificity $(82 \%)$ for detecting obstructive CAD ( $\geq 50 \%$ stenosis). ${ }^{15}$ Among patients with a normal SPECT, $14 \%$ of patients had obstructive CAD on invasive angiography. ${ }^{15}$ Marwick et al found that while the sensitivity of dipyridamole thallium scintigraphy for detecting obstructive CAD was $95 \%$ 
Table 1. Sensitivity and specificity of stress test modalities to predictive obstructive CAD in patients with CKD (potential kidney transplant recipients)

\begin{tabular}{lll}
\hline \multicolumn{1}{c}{ Stress test } & \multicolumn{1}{c}{ Sensitivity } & \multicolumn{1}{c}{ Specificity } \\
\hline Exercise stress test ${ }^{\dagger}$ & $0.36-1.00$ & $0.00-0.91$ \\
Myocardial perfusion scintigraphy & $0.74(95 \% \mathrm{Cl} 0.54-0.87)$ & $0.70(95 \% \mathrm{Cl} 0.51-0.84)$ \\
Dobutamine stress echocardiography & $0.79(95 \% \mathrm{Cl} 0.67-0.88)$ & $0.89(95 \% \mathrm{Cl} 0.81-0.94)$ \\
\hline
\end{tabular}

Data from Wang et al. ${ }^{13}$

'Data based on two studies only and no pooled estimates provided.

in non-CKD control group, it was only $37 \%$ in dialysis patients even after matching for severity and extent of CAD. ${ }^{16}$ In this study, the probability of obstructive CAD in patients with normal SPECT was $38 \%$. Finally, in a meta-analysis of nine studies of myocardial perfusion SPECT in potential kidney transplant recipients, the pooled sensitivity was $74 \%$ with a pooled sensitivity of $70 \%$, both with wide confidence intervals (Table 1$).{ }^{13}$ The reason for reduced sensitivity of myocardial perfusion SPECT in CKD/ESRD patients is not clearly known but has been attributed to a number of mechanisms ${ }^{16}$ : (a) "balanced ischemia" due to multivessel disease resulting in global ischemia. Patients with CKD have increased prevalence of multivessel disease. Since myocardial perfusion SPECT is based upon comparison of perfusion between myocardial segments, balanced multivessel disease can potentially result in false-negative SPECT; (b) presence of collateral vessel that may prevent the establishment of zones of disparate flow thereby producing the appearance of homogeneous perfusion and a false-negative SPECT; and (c) higher resting levels of adenosine (resulting in higher resting coronary flow) seen in patients with ESRD may result in reduced vascular responsiveness to dipyridamole resulting in "inadequate stress."

\section{ANCILLARY STRESS TEST MARKERS OF HIGH RISK}

Given the limitations of myocardial perfusion SPECT in CKD cohort, there is a need for ancillary markers to improve its accuracy. In this issue of the journal, AlJaroudi et al evaluate the use of one such ancillary marker-heart rate response during regadenoson stress MPI in patients with ESRD. ${ }^{17}$ Heart rate response was defined as $100 *$ (peak stress heart rate resting heart rate)/resting heart rate with values $<28 \%$ considered as a blunted heart rate response. From a cohort of 303 patients with ESRD, blunted heart rate response was associated with a twofold to threefold increased adjusted risk for all-cause mortality and also other cardiovascular endpoints and was associated with increased event rate among patients with both normal and abnormal MPI and added incremental prognostic value. ${ }^{17}$ The strengths of the analyses are the large number of patients with ESRD and the use of robust statistical techniques including propensity score matching to account for measured confounders. The limitations are the post hoc nature of the analyses and the inability to account for unmeasured confounders such as frailty. In addition, although the authors evaluated long-term outcomes, no data were presented on the presence or absence of obstructive CAD on invasive angiography. It is also not known if the increased risk seen with blunted heart rate response is merely an epiphenomenon of underlying sickness/chronicity of comorbidities and frailty and if this marker be amenable to treatment and if it can be reversed. However, heart rate response is attractive as it is simple to calculate from data which is generally collected during stress test and does not add to the cost of the stress testing.

Various other ancillary markers have been shown to identify patients at high risk of cardiovascular outcomes (Table 2). In patients undergoing exercise stress test, a plethora of prognostic data is available from exercise stress test variables. ${ }^{18} \mathrm{Few}$ of the markers of adverse prognosis include high resting heart rate, low time to peak heart rate, chronotropic incompetence (defined as inability to achieved $\geq 85 \%$ age predicted maximal heart rate, low percent heart rate reserve, or a low chronotropic index), abnormal heart rate recovery (defined as difference in heart rate between peak exercise and 1 or 2 min later), abnormal functional capacity, and exerciseinduced hypotension. ${ }^{18}$ Similarly, some of the above parameters have also been tested in patients undergoing pharmacological stress test including the heart rate response evaluated in this study and indices of chronotropic incompetence including heart rate reserve in those undergoing dobutamine stress test ${ }^{19,20}$ (Table 2). Moreover, the EKG parameters of ischemic ST-T changes during stress or recovery and ventricular arrhythmia during stress testing have been shown to have prognostic value. ${ }^{18}$ Ancillary markers of high risk for patients undergoing SPECT included increased lung 


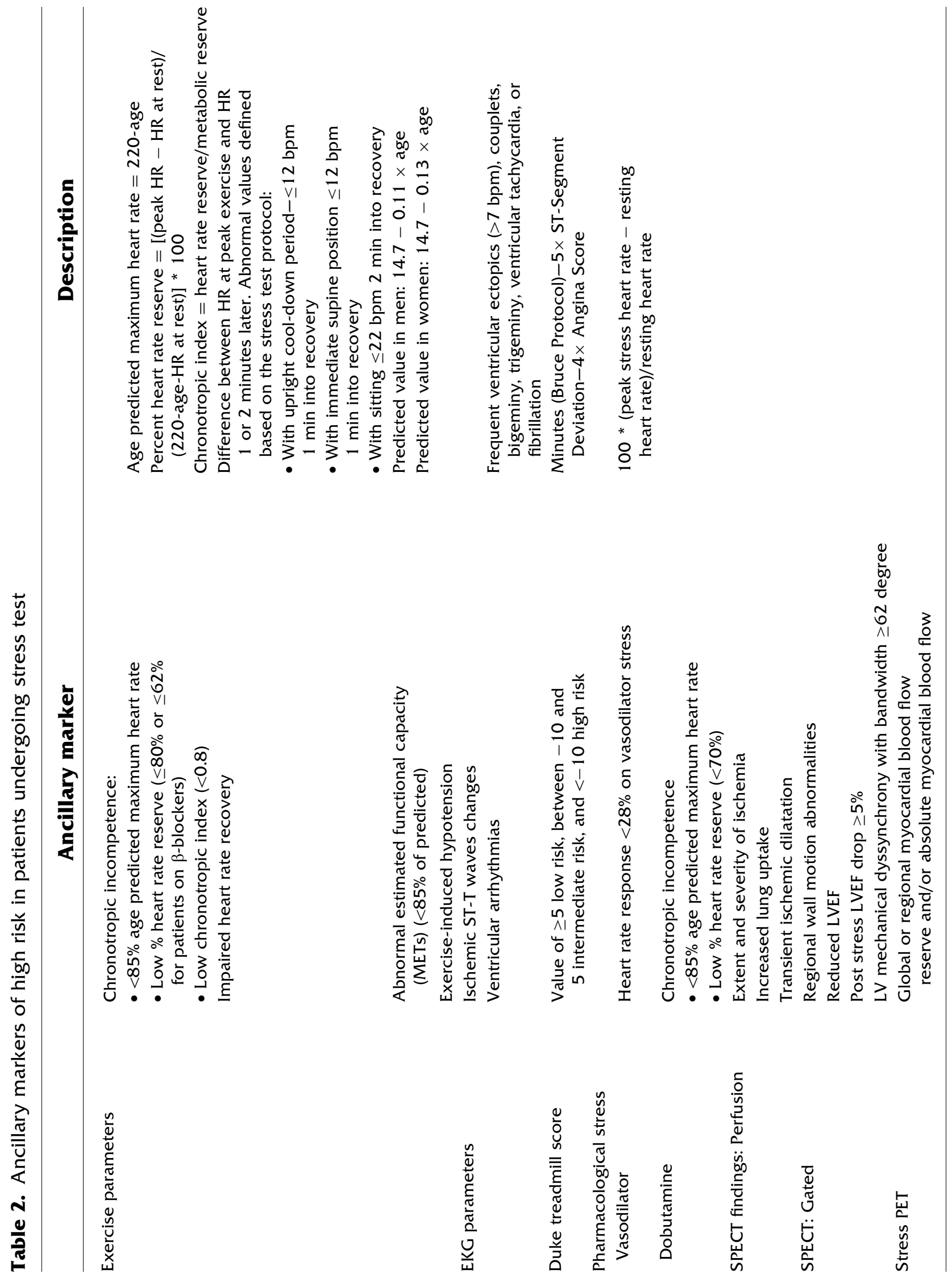


uptake and transient ischemic dilatation, both of which have been shown to be markers for extensive and severe CAD including multivessel disease, proximal left anterior descending artery disease or left main coronary artery disease. ${ }^{21}$ In addition, additional prognostic information can be gleaned from gated SPECT including information on regional wall motion abnormalities and left ventricular ejection fraction. Thus, there is a plethora of ancillary markers to identify patients at high risk of future cardiovascular events. Although many of the above ancillary markers have not been tested in the CKD/ESRD cohort and further studies are needed, given the low sensitivity and specificity of SPECT in this cohort, it is perhaps reasonable to use these ancillary markers for better risk stratification and prognostication of this high-risk group of patients. In addition to stress test variables, clinical variables such as age, duration of dialysis, diabetes, typical angina, and pre-test probability have been used as other ancillary markers of high risk in patients with CKD/ESRD. While it is not clear as to whether the various ancillary markers indicate presence of CAD in those with normal myocardial perfusion SPECT and if they should be referred for invasive angiography, it perhaps is helpful in patients with some ischemia on myocardial perfusion SPECT. The presence of ancillary markers may place such patients at a higher risk with considerations for more aggressive management including invasive angiography.

Although the above parameters identify patients with high risk, it is not known how best to treat such high-risk patients identified during stress testing. While several randomized trials in the past decade have evaluated revascularization or medical therapy alone for patients with stable ischemic heart disease, the majority of these trials have largely excluded patients with CKD/ESRD or included a very small proportion of these patients. ${ }^{22,23}$ The management of patients with CKD/ESRD has been extrapolated from data in the nonCKD cohort. It is not unknown if this extrapolation is justifiable. The International Study of Comparative Health Effectiveness with Medical and Invasive Approaches-Chronic Kidney Disease (ISCHEMIA-CKD) is an international comparative effectiveness study to determine the best approach to manage stable ischemic heart disease in patients with advanced chronic kidney disease (eGFR $<30$ or on dialysis). ${ }^{24}$ Patients will be randomized after an abnormal stress test to either an invasive strategy of cardiac catheterization with optimal revascularization and optimal medical therapy or to a conservative strategy of optimal medical therapy alone with cath reserved for OMT failures. The trial will provide important insights into the management of this high-risk group of patients.
In conclusion, the sensitivity and specificity of stress testing (including myocardial perfusion SPECT) are lower in subjects with CKD/ESRD when compared with non-CKD cohorts. A wealth of ancillary markers are available from a stress test which perhaps can be used to better risk stratify and prognosticate such patients.

\section{Acknowledgments}

Dr Bangalore reports NIH grant support for the ISCHEMIA-CKD trial.

\section{Disclosure}

This article refers to work supported by National Heart, Lung, and Blood Institute grant U01HL117905. The content is solely the responsibility of the author and does not necessarily represent the official views of the National Heart, Lung, and Blood Institute or the National Institutes of Health.

\section{References}

1. U.S. Renal Data System. USRDS 2011 annual data report: Atlas of chronic kidney disease and end-stage renal disease in the United States. 2011.

2. Wild S, Roglic G, Green A, Sicree R, King H. Global prevalence of diabetes: Estimates for the year 2000 and projections for 2030. Diabetes Care 2004;27:1047-53.

3. Couser WG, Shah S, Kopple J, Beerkens P, Wilson A, Feehally J, et al. A call to action on world kidney day, 8 March 2007. Kidney Int 2007;71:369-70.

4. Foley RN, Parfrey PS, Sarnak MJ. Clinical epidemiology of cardiovascular disease in chronic renal disease. Am J Kidney Dis 1998;32:S112-9.

5. Tonelli M, Wiebe N, Culleton B, House A, Rabbat C, Fok M, et al. Chronic kidney disease and mortality risk: A systematic review. J Am Soc Nephrol 2006;17:2034-47.

6. Go AS, Chertow GM, Fan D, McCulloch CE, Hsu CY. Chronic kidney disease and the risks of death, cardiovascular events, and hospitalization. N Eng1 J Med 2004;351:1296-305.

7. Hachamovitch R, Berman DS, Kiat H, Cohen I, Cabico JA, Friedman J. Exercise myocardial perfusion spect in patients without known coronary artery disease: Incremental prognostic value and use in risk stratification. Circulation 1996;93:905-14.

8. Antman EM, Anbe DT, Armstrong PW, Bates ER, Green LA, Hand M, et al. ACC/AHA guidelines for the management of patients with st-elevation myocardial infarction-Executive summary: A report of the American College Of Cardiology/American Heart Association task force on practice guidelines (writing committee to revise the 1999 guidelines for the management of patients with acute myocardial infarction). Circulation 2004;110:588-636.

9. Hyre AD, Fox CS, Astor BC, Cohen AJ, Muntner P. The impact of reclassifying moderate $\mathrm{CKD}$ as a coronary heart disease risk equivalent on the number of us adults recommended lipid-lowering treatment. Am J Kidney Dis 2007;49:37-45.

10. Kasiske BL, Guijarro C, Massy ZA, Wiederkehr MR, Ma JZ. Cardiovascular disease after renal transplantation. J Am Soc Nephrol 1996;7:158-65. 
11. Schmidt A, Stefenelli T, Schuster E, Mayer G. Informational contribution of noninvasive screening tests for coronary artery disease in patients on chronic renal replacement therapy. Am J Kidney Dis 2001;37:56-63.

12. De Lima JJ, Sabbaga E, Vieira ML, de Paula FJ, Ianhez LE, Krieger EM, et al. Coronary angiography is the best predictor of events in renal transplant candidates compared with noninvasive testing. Hypertension 2003;42:263-8.

13. Wang LW, Fahim MA, Hayen A, Mitchell RL, Baines L, Lord S, et al. Cardiac testing for coronary artery disease in potential kidney transplant recipients. Cochrane Database Syst Rev 2011;2011:CD008691.

14. Sharma R, Pellerin D, Gaze DC, Gregson H, Streather CP, Collinson PO, et al. Dobutamine stress echocardiography and the resting but not exercise electrocardiograph predict severe coronary artery disease in renal transplant candidates. Nephrol Dial Transpl 2005;20:2207-14.

15. Winther S, Svensson M, Jorgensen HS, Bouchelouche K, Gormsen LC, Pedersen BB, et al. Diagnostic performance of coronary CT angiography and myocardial perfusion imaging in kidney transplantation candidates. JACC Cardiovasc Imaging 2015;8:553-62.

16. Marwick TH, Steinmuller DR, Underwood DA, Hobbs RE, Go RT, Swift C, et al. Ineffectiveness of dipyridamole SPECT thallium imaging as a screening technique for coronary artery disease in patients with end-stage renal failure. Transplantation 1990;49:100-3.

17. AlJaroudi W, Campagnoli T, Fughhi I, Wassouf M, Ali A, Doukky R. Prognostic value of heart rate response during regadenoson stress myocardial perfusion imaging in patients with end stage renal disease. J Nucl Cardiol 2015. doi:10.1007/s12350-015-0234-0.
18. Kligfield P, Lauer MS. Exercise electrocardiogram testing: Beyond the ST segment. Circulation 2006;114:2070-82.

19. Bangalore S, Shah A, Vragel S, Weinberg C, Garcia W, Sawhney $\mathrm{S}$, et al. Chronotropic incompetence in patients on beta blocker referred for stress echocardiography: Superiority of heart rate reserve over $85 \%$ maximum predicted heart rate. J Am Coll Cardiol 2006;47:133A.

20. Bangalore S, Yao SS, Chaudhry FA. Comparison of heart rate reserve versus $85 \%$ of age-predicted maximum heart rate as a measure of chronotropic response in patients undergoing dobutamine stress echocardiography. Am J Cardiol 2006;97:742-7.

21. Abidov A, Bax JJ, Hayes SW, Hachamovitch R, Cohen I, Gerlach $\mathrm{J}$, et al. Transient ischemic dilation ratio of the left ventricle is a significant predictor of future cardiac events in patients with otherwise normal myocardial perfusion SPECT. J Am Coll Cardiol 2003;42:1818-25.

22. Boden WE, O'Rourke R, Teo K, Hartigan P, Maron D, Kostuk W, et al. Courage trial research group. Optimal medical therapy with or without PCI for stable coronary disease. N Engl J Med 2007;15:1503-16.

23. BARI 2D Study Group, Frye RL, August P, Brooks MM, Hardison $\mathrm{RM}$, Kelsey SF, et al. A randomized trial of therapies for type 2 diabetes and coronary artery disease. N Engl J Med 2009;360:2503-15.

24. ClinicalTrials.gov. Ischemia-chronic kidney disease trial. 2015. 